\title{
Dental Identification Using Dental Cone-Beam Computed Tomography
}

\author{
Akiko Kumagai $^{1 *}$, Akira Fujimura ${ }^{2}$ and Koji Dewa ${ }^{3}$
}

${ }^{1}$ Doctor of Dental Surgery, Division of Oral and Maxillofacial Surgery, Department of Reconstructive Oral and Maxillofacial Surgery, School of Dentistry, Iwate Medical University, Japan

${ }^{2}$ Professor, Division of Functional Morphology, Department of Anatomy, Iwate Medical University, Japan

${ }^{3}$ Professor, Department of Legal Medicine, Iwate Medical University, Japan

\begin{abstract}
In recent years, understanding three-dimensional (3D) structures has become necessary for advanced dental treatments. Therefore, dental cone-beam computed tomography (CBCT) is considered to be a very effective method. In this study, we evaluated the possibility of using СBCT to identify individuals using dental records. We first conducted CBCT on a dry skull with remaining teeth, restorations and prostheses and confirmed that the highest tube voltage of $90 \mathrm{~V}$ and tube current of $2.5 \mathrm{~mA}$ optimally detected intraoral restorations and prostheses, maximally inhibiting metal artifact. Thereafter, we performed CBCT of the soft tissue attached to the head of cadavers used for anatomical practice by dental students of our university under the same conditions. Dental charts were produced based on those obtained by visual observation and were compared with the 3D images that were constructed using analytical software. The presence of restorations and prostheses were confirmed, and the 3D morphology, which is difficult to confirm using simple X-ray images, was understood and mostly agreed with the results from visual observation. These results suggested the usefulness of CBCT in the dental examination of dead bodies when opening the mouth is impossible and suggested the possibility of yielding as one of the screening method when investigating unidentified bodies.
\end{abstract}

Keywords: Forensic dentistry; Forensic odontology; Dental identification; Dental cone-beam computed tomography; 3-D imaging; Dental restoration; Dental prosthesis

\section{Introduction}

In recent years, understanding three-dimensional (3D) structures is necessary for advanced dental imaging and treatment. Dental conebeam computed tomography (CBCT) is considered to be a very effective method. Compared with general medical CT, its advantages include a high image resolution and the need for a small installation area. This apparatus was originally used to diagnose pathological changes in the jaw-bone and examine morphology before orthodontic treatments, impacted-tooth extractions, and dental implant treatments. In this study, we evaluated the possibility of applying CBCT to dentally identify individuals. This study may be possible to use in the event of large-scale disasters with many casualties, dental identification of victims can be made by dentists not only with the postmortem dental records, but also can be used for identification of bodies through comparison of ante mortem data.

\section{Materials and Methods}

\section{Apparatus}

A dental CBCT apparatus in the Dental Treatment Center, Iwate Medical University Hospital was used (3D Accuitomo, J Morita Manufacturing Corp., Kyoto, Japan). In conventional medical or current multi-slice CT, the X-ray beam is in a fan form and the X-ray tube faces the one-dimensional detector. In $\mathrm{CBCT}$, the $\mathrm{X}$-ray tube faces the two-dimensional (2D) detector, and the X-ray beam is in a conical form to obtain 2D data. Therefore, 3D images can be created with one scan, and the 3D data can be rapidly constructed. Accurate images can be created with isotropic voxels of at least approximately $0.1 \mathrm{~mm}$, and its spatial resolution is marked with an equivalent resolution in the axial direction [1]. Furthermore, the present apparatus can be installed in a similar space as panoramic X-ray apparatuses. However, because the X-ray scatter is high and the density resolution is low in CBCT, diagnosing soft tissue diseases is difficult. Quantitative analysis of ossification changes is impossible because the image density obtained by CBCT cannot be converted into $\mathrm{CT}$ values (X-ray attenuation coefficient) to quantify $\mathrm{X}$-ray transmittance using relative values $[1,2]$.
3D images were constructed with the CBCT data obtained using analytical software (Zed View, LEXI, Co. Ltd., Tokyo, Japan).

\section{Materials}

We used maxillary and mandibular dry skulls with remaining teeth, restorations, and prostheses to confirm the restorations, and prostheses in 7 cadavers that are used for anatomical practice by the dental students of our university. Formalin fixed skulls, which were unable to open their mouths, were used for the CT photography of unknown oral restorations and prostheses.

\section{Methods}

We conducted CBCT on a dry skull. We adjusted the window level and window range, detected only the dental prostheses for visual estimation and created 3D images. Thereafter, we performed CBCT of the soft tissue attached to the heads of cadavers under the same conditions. Then, 3D images were constructed using analytical software (Figure 1). It is difficult to detect acrylic resin base dentures simply by adjusting the window level and window range. For the dentures, we performed an image elimination treatment of the surrounding tissue to detect the outline of dentures and constructed $3 \mathrm{D}$ images using analytical software. Among the 7 cadavers, 3D images of 4 cadavers were compared with dental chart findings obtained by visual observation. Four observers produced the dental charts after the median of the head was sectioned for the internal observation during anatomical practice. Thereafter, we evaluated the correspondence

*Corresponding author: Akiko Kumagai, PhD, Division of Oral and Maxillofacial Surgery, Department of Reconstructive Oral and Maxillofacial Surgery, School of Dentistry, Iwate Medical University, 19-1, Uchimaru, Morioka, Iwate, 020-8505, Japan, Tel: +81-19-6515111 (4512); Fax: +81-19-623-6757; E-mail: kumagaia@iwate-med.ac.jp

Received August 06, 2015; Accepted August 28, 2015; Published September 08, 2015

Citation: Kumagai A, Fujimura A, Dewa K (2015) Dental Identification Using Dental Cone-Beam Computed Tomography. Dentistry 5: 332. doi:10.4172/21611122.1000332

Copyright: ( $) 2015$ Kumagai A, et al. This is an open-access article distributed under the terms of the Creative Commons Attribution License, which permits unrestricted use, distribution, and reproduction in any medium, provided the original author and source are credited. 

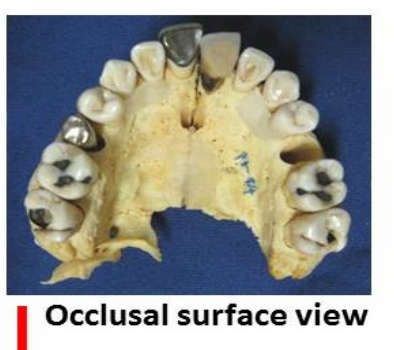

Perform CBCT

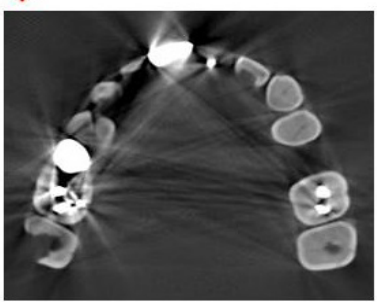

2D image

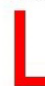

Adjustment of window level and window range

The highest tube voltage of $90 \mathrm{~V}$ and tube current of $2.5 \mathrm{~mA}$ were the optimal CBCT conditions for detecting intraoral restorations and prostheses while maximally inhibiting metal artifact.

Figure 1: Construction of a 3D image.

of restorations and prosthetic morphology between the 3D images and dental chart findings and the possibility of collecting additional anatomical information.

\section{Results and Discussion}

We confirmed that the highest tube voltage of $90 \mathrm{~V}$ and tube current of $2.5 \mathrm{~mA}$ were the optimal CBCT conditions to detect intraoral restorations and prostheses while maximally inhibiting metal artifact.

Figure 2 shows dry skull 1 , and the 3D construction and panoramic images of restorations and prostheses were obtained from CBCT. Although the resin in the cast crown in tooth 11, full cast crown in tooth 14 , amalgam filling in tooth 16 , post for root canal in tooth 21 , resin filling of the cervical region in tooth 22 , and amalgam filling in tooth 26 were confirmed, the resin jacket crown in tooth 21 and resin filling of the cervical region in tooth 12,13 and 23 could not be detected. Although the full cast crown and dummy of the fixed dental prosthesis in a mandibular right molar and amalgam filling in tooth 37 were confirmed, the soldering metal of the fixed dental prosthesis and resin of dummy in tooth 46 were not detected.

Figures 3, 4 and 5 show the 3D construction and panoramic images of prostheses obtained from the CBCT of each skull of cadavers for anatomical practice. Figure 3 shows that in the cadaver (number 1 ), the resin facing the cast crown and the full cast crown, the resin filling material, and the root canal filling material could be confirmed. However, the area of the faced resin could be clearly detected. As seen in Figure 4, a partial denture was inserted into the upper jaw of the cadaver (number 2), and the clasps and wires for enforcement were confirmed. In Figure 5 (cadaver number 3), we confirmed that it was a full denture and that the denture-base shape was also possible.

Figure 6 shows the dental charts for one of the 4 cadavers used for anatomical practice produced by the 4 observers and 3D CT construction and panoramic images of the prostheses obtained from the CBCT of each cadaveric skull. This cadaver showed metal restorations and prostheses consistent with the dental chart, and root canal filling materials were also confirmed. However, some types of
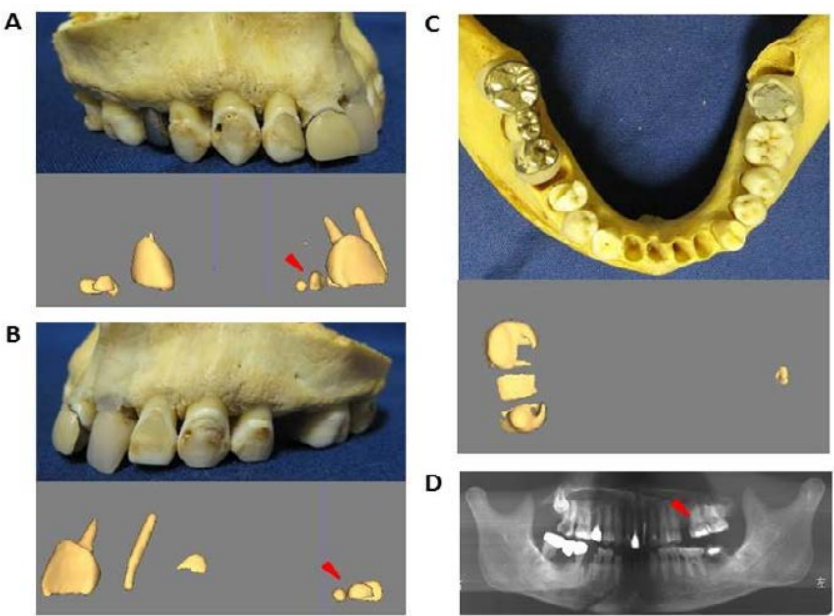

A) Right side of Upper jaw; B) Left side of Upper jaw; This dry skull is the same as C) Occlusal surface side of the lower jaw ; D) Panoramic radiograph. Arrow heads are an amalgam filling of tooth 26.

Figure 2: A, B and C contain the photograph (top) and 3D images (below) of the dry skull with the remaining teeth, restorations, and prostheses.
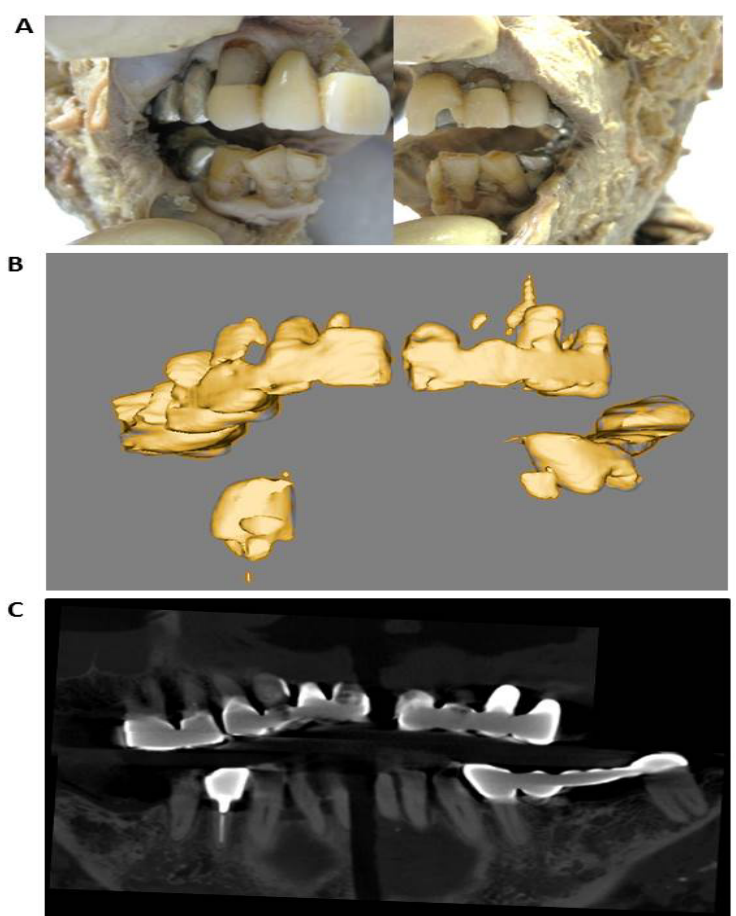

A) Extra-oral finding; B) 3D image of front view; C) 2D image. The resin faced cast crown, full cast crown, resin filling material, and root canal filling material could be confirmed. However, the area of faced resin could be clearly detected.

Figure 3: Cadaver (No. 1) for anatomical practice used by the dental students. 
A

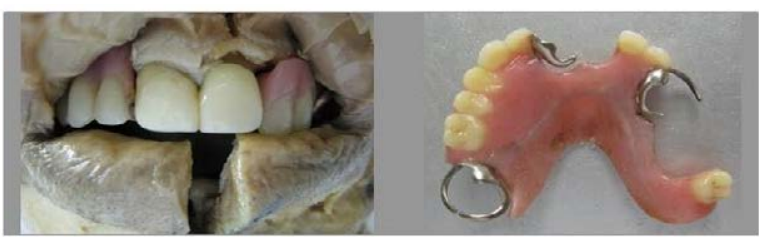

B
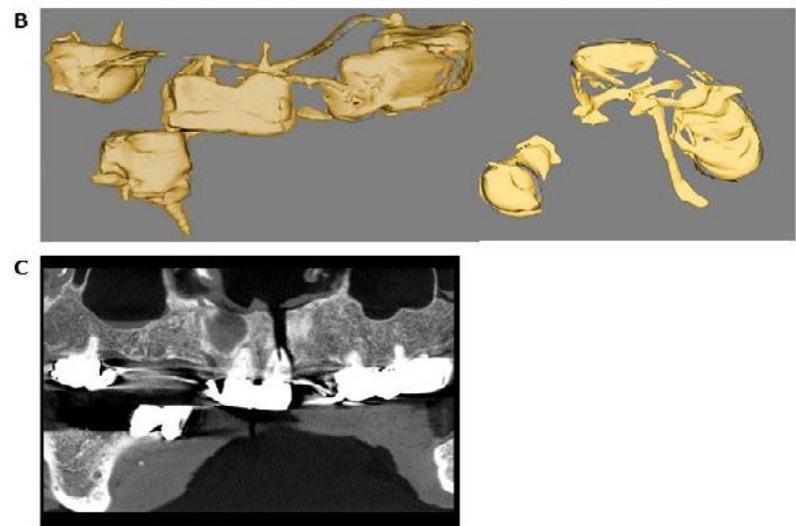

A) Extra-oral finding (on the left) and partial denture (on the right); B) 3D image of the front view (on the left) and occlusal surface view (on the right); C) 2D image. A partial denture was inserted in the upper jaw, and the clasps and wires for enforcement were confirmed.

Figure 4: Cadaver (No. 2) for anatomical practice used by the dental students.

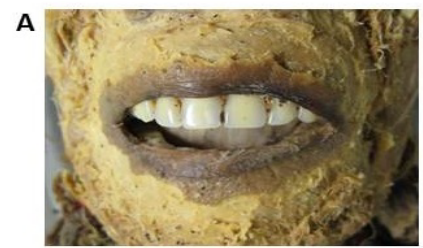

B
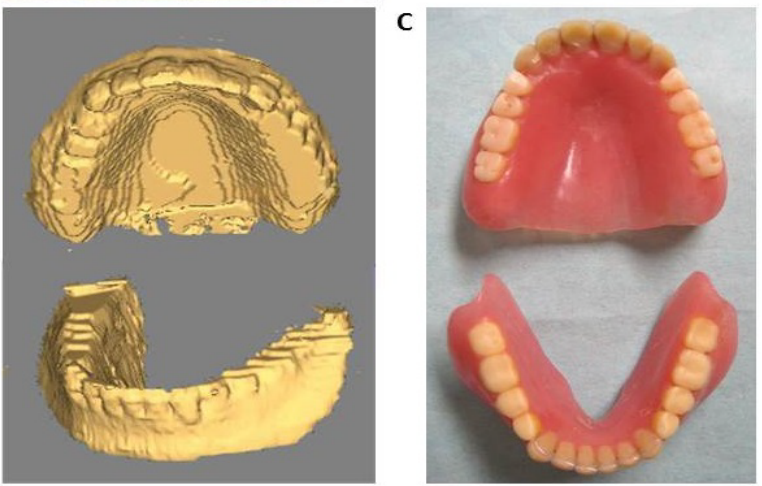

A) Extra-oral image; B) 3D image of the full denture; C) Photograph of the full denture. For the dentures, we performed the image elimination treatment of the surrounding tissue to detect the outline of dentures and constructed 3D images using analytical software.

Figure 5: Cadaver (No. 3) for anatomical practice used by the dental students.

composite resin could be detected, and some could not. In addition, the porcelain was clearly detected.

As a result, the presence of restorations and prostheses was readily confirmed, and the 3D morphology, difficult to confirm using simple
X-ray images, could be understood. From these results, dental CBCT readily confirmed the presence of restorations and prostheses, root canal filling materials, and denture clasps and wires for enforcement in both dry and soft tissue-attached skulls. The 3D morphology, such as in full dentures, was difficult to confirm using simple X-ray images but could be understood using CBCT. Furthermore, the 3D images obtained by dental CBCT did not contradict the dental records. However, detecting the composite resin was difficult depending on its type, and the outline of the restorations could not be clearly detected when the contaminants were attached. Because of the artifact changes depending on the type of metal, uniform detecting was difficult in some cases. Because porcelain develops artifact differently than metal, the simultaneous detecting of porcelain and other metals was impossible using window level adjustment at the time of the $3 \mathrm{D}$ reconstruction.

In severely burned or decomposed bodies, comparing the personal identification using dental findings from bodies and dental materials with dental records and X-ray photographs is effective. However, collecting dental findings is frequently difficult due to the impossibility of mouth opening and contamination by the environment in which bodies are found. In particular, in the identification of bodies with severe damage, such as with victims of large-scale disasters, rapid personal identification is required. In recent years, there have been many reports regarding the usefulness of autopsy imaging in which CT and MRI are conducted before forensic autopsy [3-5]. The primary advantage of this method is that it is non-destructive and avoids the destruction of bodies, including the face. Furthermore, to efficiently detect information, various visualization methods have been applied. A report utilizing a digital image also appears in the Forensic Odontology field [6-11]. However, CT images are frequently not useful for intraoral observation due to the occurrence of metal artifacts. On the other hand, although the X-ray photographable range of the dental
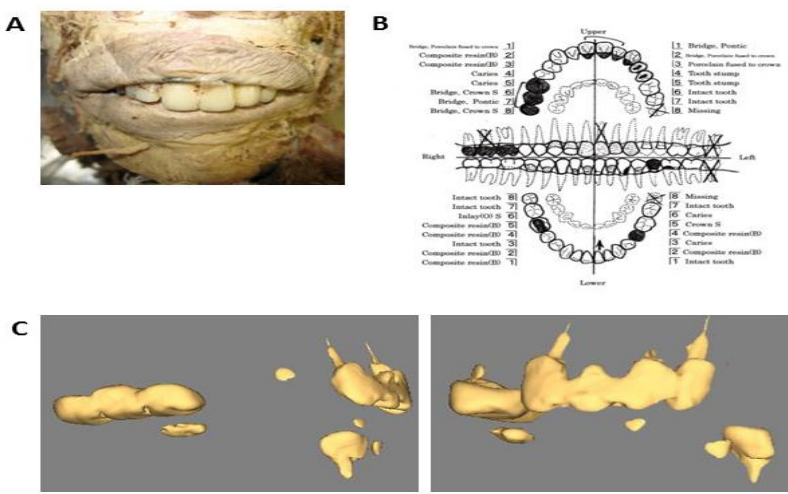

D

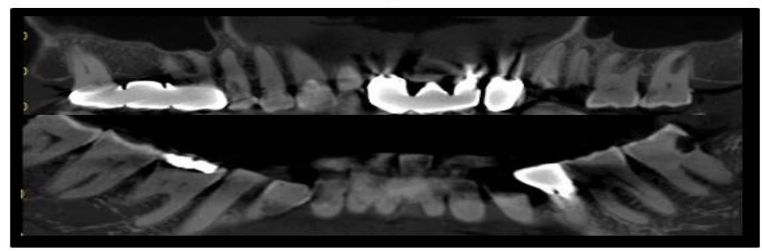

A) Extra-oral finding; B) Dental chart that the 4 observers produced; C) 3D image of the right side view (on the left) and left side view (on the right); D) $2 \mathrm{D}$ image. Metal restorations and prosthesis did not contradict the dental chart; root canal filling materials also confirmed the findings. However, some types of composite resin can be detected, and some cannot. In addition, porcelain could be clearly detected.

Figure 6: Cadaver (No. 4) for anatomical practice used by the dental students. 
CBCT used in this study is narrow, the X-ray range is sufficient for intraoral observation. CBCT also succeeded in detecting restorations and prostheses, root canal filling materials, and dentures as images by processing them on a personal computer display that utilizes metal artifacts, which is a disadvantage of CT images. СBCT enables the collection of dental findings in areas that are difficult to observe; combined with visual observation, it is possible to detect the composite resin, which is overlooked due to contamination, depending on the type of composite resin. This has been suggested to be a markedly effective auxiliary means to collect materials.

In Japan, although marking at the time of prosthesis production has been evaluated for identification, the morphology, material quality, and intraoral durability of the prostheses are problematic. If image processing, can be used, such as in this study, it is expected that the mark inscription method will spread in the future. Although the usefulness of personal identification by inscribing marks on the denture surface has been reported [12], personal identification on images is possible only by inserting personal information into the denture bases, using metals, of the denture wearers by the dentists producing the dentures.

Practical apparatuses for dental CBCT were only developed 14 years ago and have been gradually adopted in the clinical field. Therefore, practical use in dental forensics is not yet realistic. However, the results of this study suggest the usefulness of CBCT to dentally examine dead bodies when opening the mouth is impossible and the possibility of yielding information for screening to investigate unidentified bodies with dental charts and 2D images. Panoramic X-ray equipment that is capable of taking photographs in supine positions has already been put into practice in forensic odontology. However, to use it in disasterstricken areas, such as those affected by the Eastern Japan earthquake and tsunami, we are still faced with many challenges, such as the problem of radiation exposure and the difficulty of coping with power failures. We expect the usefulness of CBCT to be further evaluated and that CBCT apparatuses will be improved and effectively used for dental identification. And these data to be useful a central data-pool with all dental work perform should be available, in order to match the data to a certain patient.

\section{Conclusion}

We evaluated the possibility of using CBCT for the dental identification of individuals. As a result, the presence of restorations and prostheses was readily confirmed and the 3D morphology, which is difficult to confirm using simple X-ray images, could be understood and mostly agreed with the results obtained by visual observation. These results suggest the usefulness of CBCT in the dental examination of dead bodies when mouth opening is impossible and the possibility of yielding information for screening by investigating unidentified bodies.

\section{Acknowledgments}

Special thanks to Prof. Shozushima M. (Division of Dental Radiology, Department of Reconstructive Oral and Maxillofacial Surgery, Iwate Medical University) for technical advice and the radiation engineers of the Central Radiology, Iwate Medical University Hospital and the 2008 sophomore class of School of Dentistry for their kind cooperation.

\section{References}

1. Okano T, Harata Y, Sugihara Y, Sakaino R, Tsuchida R, (2009) Absorbed and effective dose from cone-beam volumetric imaging for implant planning. Dentomaxillofac Radiol 38: 79-85.

2. Arai Y, Tammisalo E, Iwai K, Hashimoto K, Shinoda K (1999) Development of a computed tomographic apparatus for dental use. Dentomaxillofac Radiol 28 : 245-248.

3. Hayakawa M, Yamamoto S, Motani H, Yajima D, Sato Y, et al. (2005) Does imaging technology overcome problems of conventional postmortem examination? A trial of computed tomography imaging for postmortem examination. Int J Legal Med 120: 24-26.

4. Pohlenz $P$, Blessmann M, Oesterhelweg L, Habermann CR, Begemann PGC et al. (2008) 3D C-cam as an alternative modality to CT in postmortem imaging technical feasibility. Forensic Sci Int 175: 134-139.

5. O'Donnell C, lino M, Mansharan K, Leditscke J, Woodford N, et al. (2011) Contribution of postmortem multidetector CT scanning to identification of the deceased in a mass disaster: Experience gained from the 2009 Victorian bushfires. Forensic Sci Int 205: 15-28.

6. Yang F, Jacobs R, Willems G (2006) Dental age estimation through volume matching of teeth imaged by cone-beam CT. Forensic Sci Int 159: S78-S83.

7. Jackowski C, Lussi A, Classens M, Kilchoer T, Bolliger S, et al. (2006) Extended CT scale overcomes restoration caused streak artifacts for dental identification in CT-3D color encoded automatic discrimination of dental restorations. J Comput Assist Tomogr 30: 510-513.

8. Jackowski C, Wyss M, Persson A, Classens M, Thali MJ, et al. (2008) Ultrahight-resolution dual-source CT for forensic dental visualization- discrimonation of ceramic and composite fillings. Int J Legal Med 122: 301-307.

9. Bassed RB, Hill AJ (2011) The use of computed tomography (CT) to estimate age in the 2009 Victorian Bushfire Victims: A case report. Forensic Sci Int 205: 48-51.

10. Birngruber CG, Obert M, Ramsthaler F, Kreutz K, Verhoff MA et al. (2011) Comparative dental radiographic identification using flat panel CT. Forensic Sci Int 209: e31-e34

11. Woisetschläger M, Lussi A, Persson A, Jackowski C (2011) Fire victim identification by post-mortem dental CT: radiologic evaluation of restorative materials after exposure to high temperatures. Eur J Radiol 80: 432-440.

12. Naito Y, Meinar AN, Iwawaki Y, Kashiwabara T, Goto T, et al. (2013) Recording of identification information on dental prostheses using fluorescent material and ultraviolet light. Int J Prosthodont 26: 172-174. 